\title{
Prácticas profesionales durante la formación inicial docente: análisis y optimización de sus aportes a los que aprenden y a los que enseñan a aprender "a enseñar"
}

\author{
Professional practices in initial Teacher education: analysis and optimization of its \\ contributions to those who learn and to those who teach to learn "to teach"
}

Práticas profissionais durante a formação docente inicial: análise e otimização de suas contribuições para aqueles que aprendem e aqueles que ensinam a aprender "a ensinar"

\author{
José Cornejo Abarca \\ Pontificia Universidad Católica de Chile, Facultad de Educación \\ Telf.: 56 22719787. Correo electrónico: jcorneja@uc.cl
}

\begin{abstract}
RESUMEN
El autor comienza por abordar el marco conceptual y operativo de prácticas profesionales durante la formación inicial docente, así como de los roles y del desarrollo profesional de los actores que intervienen en los distintos contextos de educación escolar. Se apoya en las pistas y conclusiones de reciente literatura especializada nacional e internacional y en su trayectoria profesional y académica en este campo. A partir de ello presenta y analiza políticas y prácticas institucionales implementadas; valora críticamente diversos aportes conceptuales y prácticos. Finalmente, sugiere propuestas que apunten a optimizar las transformaciones requeridas en las prácticas profesionales, en la perspectiva de un cambio progresivo, integral y coherente de la calidad de la Formación inicial docente en el sistema educacional chileno.

Palabras clave: formación inicial docente, prácticas profesionales, enfoques y dispositivos, actores y roles, formación de profesores y de formadores.
\end{abstract}

\section{ABSTRACT}

The author begins by addressing the conceptual and operational framework of professional practice for initial teacher education, as well as the roles and professional development of actors involved in the different contexts of schooling. It relies on the slopes and conclusions of recent national and international literature and in his professional and academic careers in this field. From this, presents and analyzes the implemented policies and practices; and critically evaluates various conceptual and practical contributions. Finally, suggests proposals aimed at optimizing the required transformations in professional practices, with a view to a progressive, comprehensive and consistent change in the quality of initial teacher training in the Chilean educational system.

Key words: initial teaching education, professional practices, approaches and dispositives, actors and roles, education of teachers and teachers educators.

\section{RESUMO}

Inicia-se abordando o quadro conceitual e operacional de práticas profissionais durante a formação docente inicial, bem como os papéis e o desenvolvimento profissional dos atores envolvidos nos diversos contextos da educação escolar. Baseia-se em pistas e conclusões de recentes e especializadas literaturas nacional e internacional e em sua trajetória profissional e acadêmica nesta área. A partir daí, apresentam-se e analisam-se políticas e práticas 
Estudios Pedagógicos, vol. XL, Número Especial 1: 239-256, 2014

PRÁCTICAS PROFESIONALES DURANTE LA FORMACIÓN INICIAL DOCENTE: ANÁLISIS Y OPTIMIZACIÓN DE SUS APORTES A LOS QUE APRENDEN Y A LOS QUE ENSEÑAN A APRENDER "A ENSEÑAR"

institucionais implementadas; avaliam-se criticamente diversas contribuições conceituais e práticas. Finalmente, sugerem-se propostas que sinalizam a otimização das transformações necessárias às práticas profissionais, na perspectiva de transformação progressiva, integral e coerente com a qualidade da formação docente inicial no sistema educacional chileno.

Palavras-chave: formação docente inicial, práticas profissionais, enfoques e dispositivos, atores e papéis, formação de professores e de formadores.

\section{INTRODUCCIÓN}

En este trabajo, procuraré compartir algunos aprendizajes que he ido logrando respecto a la compleja y desafiante tarea de formar profesores, especialmente en su formación inicial, en la cual he tenido una larga trayectoria de estudio, actuación e indagación. Cuando corresponda, lo corroboraré y complementaré con los aportes de otros profesores y académicos con quienes he trabajado y con la base de conocimientos sistematizada en la literatura especializada a la que he podido acceder.

Mi experiencia fundamental y más frecuente ha tenido como campo principal de actuación la formación en el sistema escolar y el de educación superior formales e institucionalizados. Considero que experiencias y prácticas en educación y formación menos formales e institucionalizadas, que también están operando en nuestro medio, me han aportado y podrían aportarnos potentes alternativas y complementos a nivel conceptual y operativo; no obstante aquí no lo abordaré.

$\mathrm{Al}$ interior de ese complejo y constante proceso de interacciones entre sujetos que caracteriza la formación de profesores, mi foco se centrará en el dispositivo correspondiente a las prácticas profesionales durante la formación inicial, procurando no desconocer sus interacciones con otros dispositivos del programa formativo. Para apreciar más concretamente la complejidad y las dinámicas implicadas en lo recién descrito, me he planteado las siguientes interrogantes que orientan mi análisis y propuestas:

- ¿Desde dónde, por qué y para qué se ha llegado recientemente al componente de las "prácticas profesionales" en la formación inicial de futuros profesores?, ¿qué fundamentos y supuestos han influido en la modalidad que éstas han adquirido?

- ¿Qué "actores" han ido adquiriendo protagonismo conceptual y práctico en su implementación y ejecución? ¿Qué roles se han ido perfilando respecto de los actores identificados, ya sea por asignación y/o apropiación?; ¿cuáles se destacan especialmente acerca de los que tienen la tarea de "formar para aprender a enseñar"?

- ¿Qué adquisiciones conceptuales y operativas se han podido identificar y, en alguna medida, destacar y evidenciar como factores a considerar en el cambio y la transformación de la calidad de las actividades de "prácticas"?

\section{DISCURSOS Y SUPUESTOS CONCEPTUALES SOBRE LAS PRÁCTICAS PROFESIONALES DE LA FORMACIÓN INICIAL DOCENTE.}

No es novedad que educar, enseñar y formar implican necesariamente componentes de actuación, ejecución, puesta en práctica y ello ha formado parte de toda experiencia 
histórica de la educación. Lo que no ha sido igualmente considerado críticamente y a veces concebido como necesario o natural en dicha actuación es, entre otros, el significado y sentido de dimensiones tales como su finalidad, calidad, contextos, saberes y posiciones del o los actores de ésta.

\subsection{ENFOQUES DE RELACIONES ASIMÉTRICAS Y DUALISTAS DE LAS RELACIONES ENTRE TEORÍA Y PRÁCTICA}

Aunque no ha desaparecido del todo como medida de política o de actuación, hace un tiempo el mandato o la asignación de formación inicial para la enseñanza venían siendo entregados exclusiva o principalmente a las Normales y/o a las Universidades y sus Escuelas o Facultades de Educación. En ese contexto, el supuesto predominante ha sido que las "prácticas" estaban para ofrecer a los estudiantes una "aplicación", en situación real, de lo aprendido en dicha formación. En tal perspectiva, la enseñanza o formación aparece subvalorada, arraigándose en una noción limitada de "entrega de información", que se suele interpretar como una tarea simple o sencilla. Al contrario de los interesantes avances que se reconocen a la compleja naturaleza del aprendizaje, es contradictorio que no se haya producido, en consecuencia, una modificación proporcional a la comprensión de la naturaleza de la enseñanza o un simple o confuso reconocimiento de su complejidad. No siempre se destacan o identifican el conocimiento y las destrezas y habilidades inherentes y necesarias a desarrollar continuamente para obtener una enseñanza de calidad.

En algunos casos, se llegó a hablar también de la promoción de un nexo entre la teoría y la práctica; pero su concepción subyacente apuntaba a que los conocimientos teóricos eran adquiridos fuera del medio escolar (en ámbitos académicos) y que el aporte del establecimiento escolar se limitaba a la dimensión práctica.

En otras visiones, se ha llegado a postular, además, que las "prácticas" permitirían a los estudiantes en formación docente una demostración de su nivel de dominio de las habilidades juzgadas como esenciales para enseñar, lo que requiere ser evaluado y establecido oficialmente. Esta tarea de constituirse en "primeros garantes" de la decisión de acceso o rechazo al ejercicio de la docencia por parte de sus estudiantes ha sido mayoritariamente atribuida a los Formadores de futuros docentes. Pero su implementación no ha estado exenta de discusiones y tensiones: factores y contenidos a considerar en la evaluación; modalidades, período y duración de su aplicación; responsabilidad pertinente a los formadores de la institución académica (en Chile habría que considerar también que son de carácter público y privado) y del centro escolar que colabora, y de los estudiantes.

Los cambios paradigmáticos no son siempre masivos ni totales; más bien suelen ser progresivos -aunque a veces con una velocidad que uno desearía fuese mayor-e incluso llegan a yuxtaponerse o combinarse, cuestionando así su carácter de transformaciones reales y profundas a que su discurso aspira. En nuestro medio nacional, hemos estado escuchando y conociendo algunas propuestas que permiten analizar críticamente los diferentes enfoques y políticas implementadas, ellas me han sugerido que es conveniente preguntarse si lo que en éstas se pone en juego son cambios -o acumulación- de dispositivos o de contenidos. Desde el punto de vista de las políticas, ciertos supuestos y medidas concretas, que ofrecerían aportes innovadores sustantivos, pueden quedar en la periferia de los sistemas institucionales principales en la medida que apelan a reestructuraciones mayores que son menos compartidas o muy demandantes de energías y voluntades. 
Estudios Pedagógicos, vol. XL, Número Especial 1: 239-256, 2014

PRÁCTICAS PROFESIONALES DURANTE LA FORMACIÓN INICIAL DOCENTE: ANÁLISIS Y OPTIMIZACIÓN DE SUS APORTES A LOS QUE APRENDEN Y A LOS QUE ENSEÑAN A APRENDER "A ENSEÑAR"

Me ha parecido oportuno presentar lo más esencial de algunas contribuciones alternativas de académicos nacionales (entre muchas otras) desarrolladas a partir de contrastaciones de marcos teóricos e investigativos aportados por literatura especializada y de resultados obtenidos por ellos mediante estudios realizados en el contexto de trabajo de profesores chilenos.

Al presentar algunos de sus artículos, Sisto $(2011,2012)$, un académico de la Escuela de Psicología de la Pontificia Universidad Católica de Valparaíso, señala que su estudio se ha focalizado en identificar y analizar cambios en el trabajo docente que han venido ocurriendo, desde 1973, en el ámbito de la educación pública en Chile, particularmente a través de la implementación de la política de evaluación e incentivos al desempeño docente (2001, Ley $19.715 ; 2004$, Leyes 19.333 y 19.961). Según él, a raíz de ello los profesores han estado sujetos a una serie de reformas que buscan hacer más eficaz la función pública mediante modificaciones en la gestión, las que han traído consigo un nuevo modelo para el trabajo público:

\begin{abstract}
Ahora este debe comprenderse como un desempeño de carácter individual orientado a cumplir estándares e incrementar resultados en mediciones de indicadores establecidos por los diseñadores de las políticas... He aquí que podemos apreciar el despliegue del llamado Nuevo Management Público, un enfoque administrativo que instala a la empresa privada como el principal modelo de eficiencia y eficacia a imitar por los servicios públicos. Su implementación desafía directamente a los modos con los cuales se había construido tradicionalmente (veremos en otra cita su sentido) el vínculo entre empleador (Estado) y trabajador (empleado público). Hoy los salarios y estabilidad laboral se están haciendo crecientemente dependientes del ajuste a estándares y logro de resultados, instalándose modelos de desarrollo profesional basados en competencia individual y emprendimiento. Las políticas de evaluación e incentivo al desempeño docente para profesores del sistema público municipalizado en Chile constituyen un ejemplo de ello (Sisto, 2012: 36).
\end{abstract}

El autor señala que durante una investigación de tres años mediante "entrevistas activas" realizadas con profesores pertenecientes al sistema municipalizado pesquisó y analizó cómo ellos se han ido posicionando y construyendo sus identidades laborales en el contexto de implementación de la política específica mencionada. En la parte correspondiente a la discusión y conclusión de su estudio, Sixto sostiene lo siguiente:

Lo que aquí se ha mostrado no son simples percepciones de la política de evaluación; las formas de describir y valorar esta política dan cuenta de una disputa en torno a las identidades laborales de los docentes..., los sujetos se posicionan de un cierto modo..., disputan terrenos claves para los procesos de construcción identitaria... tales como los sistemas de juicio en virtud de los cuales las identidades laborales pueden ser problematizadas, la autoridad de quién puede decir la verdad acerca de qué es el docente y las teleologías que dan un sentido orientador al quehacer cotidiano de los docentes... Las voces docentes, adoptando formas de habla propias de las posiciones minoritarias -con menos poder-, dan cuenta de una resistencia como oposición, socavando la política, al mostrarla como limitada y subjetiva, y describiéndose como víctimas de esta. Pero, además se busca obtener voz...; tanto la noción del profesor víctima como la versión de sí como profesional libre elector tienen como efecto relevar categorías que aparecen silenciadas en la construcción de la profesión docente que se da desde la política... (estas) coinciden en mostrar al trabajo docente como una práctica situada (en períodos o décadas anteriores a 1973), de carácter relacional, realizada en espacios locales, como el aula, y orientada a la justicia social. Así, con recursos lingüísticos heterogéneos se despliegan otros criterios con los cuales debiese ser comprendido y valorado el trabajo docente, erigiendo nuevas autoridades, 
diversas a las que ha construida la política (los que tiene la experiencia de nivel versus los que tienen tiempo de preparación), otra teleología que da sentido al desarrollo profesional docente profesional docente la justicia social y democratización del conocimiento versus el profesor que se hace asesor del alcalde, como muestra de una trayectoria individual (Sisto, 2012: 45-46).

Aunque el estudio de Sisto se refiere a una muestra de profesores en ejercicio y de distintos lugares del sector municipal, me parece que su planteamiento ofrece importantes aportes conceptuales e investigativos cuya consecución, además de optimizarse, puede enriquecer nuestro conocimiento al respecto. Por ejemplo, una postura similar había sido ya formulada en otros contextos por Van Manen (2002), quien reconocía que la discusión sobre la "tarea pedagógica" de la escuela aparecía como ambigua en la medida que el segundo término se usaba habitualmente de modo diferente y en distintos contextos y discursos. A pesar de tales diferencias, él afirmaba la existencia de un interés y una constatación compartidos por autores de distintas procedencias: la perspectiva contemporánea de políticas y discursos de su época tendía a animar al profesor a centrarse "fuera" de los estudiantes que enseña y en los resultados instruccionales, la productividad, la mejora social, los sistemas de puntaje, las medidas de rendición de cuentas, las tecnologías instruccionales, etc., sin considerar que, para los profesores, tales dimensiones son ampliamente externas a sus preocupaciones cuotidianas. En cambio, el foco prioritario de los profesores tendería a estar en la complejidad de los aspectos relacionales, personales, morales y emocionales de su actuación diaria, cuando están tratando de enseñar a los niños y jóvenes a su cargo; para estos últimos, el experimentar permanentes demandas institucionales con consecuencias laborales diferentes a las personales y colectivas sin duda les pueden implicar dificultades en sus procesos identitarios y de bienestar mental.

En tal dirección, y con una orientación bastante cercana a las de Sisto y Van Manen, aporta también otras dimensiones valiosas un estudio de Reyes et al. (2010), académicos de la Universidad de Chile. En él informan "de una instancia de trabajo colectivo de profesores (académicos) y de profesores de aula, el "Taller de Educadores autores", que ha tenido como propósito rastrear el proceso de nuevas subjetividades docentes que permitan dar cuenta de las actuales condiciones en que es desempeñada la docencia, así como proponer otras formas de ser docente" (Reyes et al., 2010: 269-292).

La consideración del desarrollo y la identidad profesional como tareas más interactivas y progresivas, con un inicio más precoz que la entrada a la formación inicial y más continua que la iniciación en el ejercicio de la docencia, puede beneficiarse si se van conociendo mejor propuestas de políticas y programas que intentan abordarla. Yo sugiero considerar también las diversas formas y calidad de "modelización" que van teniendo los momentos y espacios del ejercicio de la enseñanza.

\subsection{ENFOQUES QUE APUNTAN A RELACIONES MÁS SIMÉTRICAS, SISTÉMICAS E INTERACTIVAS ENTRE TEORÍA Y PRÁCTICA}

Desde las últimas décadas del siglo pasado, se ha venido configurando e impulsando a nivel internacional un "movimiento de profesionalización de la enseñanza". Esto ha conducido a un cúmulo importante de propuestas de políticas y modalidades operativas mediante reformas y cambios de la formación de quienes la van a ejercer o están ya ejerciéndola y de quienes los forman para ello (entre otros, Ávalos, 2002; Feiman-Nemser, 
Estudios Pedagógicos, vol. XL, Número Especial 1: 239-256, 2014

PRÁCTICAS PROFESIONALES DURANTE LA FORMACIÓN INICIAL DOCENTE: ANÁLISIS Y OPTIMIZACIÓN DE SUS APORTES A LOS QUE APRENDEN Y A LOS QUE ENSEÑAN A APRENDER "A ENSEÑAR"

2001; Imbernón, 1994; Korthagen et al., 2001; Loughran et al., 2004; Ministerio de Educación [MINEDUC], 2005; Vaillant y Marcelo, 2001).

Tal perspectiva "profesional" acerca del ejercicio docente ha dado origen a una "recentración" de los dispositivos de "prácticas" durante el desarrollo de los programas de formación inicial para la enseñanza o docencia. Según Gervais y Desrosiers (2005), algunas de sus innovaciones se han manifestado en una alternancia de la formación inicial docente ofrecida tanto por la institución de educación superior como por el establecimiento escolar, y en la distribución de la responsabilidad o compromiso por parte de ambos contextos de formación. Por otra parte, han tenido que irse revisando las modalidades de trabajo conjunto y colaboración, profundizando en las exigencias de innovación de estas desde la perspectiva de la integración de los aprendizajes que aportan. A ello, hay que agregar la identificación y redefinición de los roles de la variedad de actores que participan en esta co-formación, especialmente de los que trabajan como Formadores de los estudiantes. Otra de las incidencias señaladas por las autoras canadienses mencionadas refiere a la prioridad atribuida a la formación en situación o en el terreno como uno de los componentes esenciales de todo programa de formación inicial.

En el último tiempo, y más recientemente en nuestro medio nacional, en los programas de formación inicial las prácticas de formación han ido siendo abordadas más bien como experiencias que apuntan a que los estudiantes identifiquen y aprendan una imagen realista del medio o contexto de trabajo docente y de la profesión docente como tal. Se apunta también a que las "prácticas" promuevan y faciliten el desarrollo progresivo de "competencias profesionales" en las que movilicen y pongan en juego recursos de diversa naturaleza. Hago notar que acerca de la noción destacada entre comillas no existen conceptos ni dispositivos/ modalidades de formación y evaluación que encuentren similar aceptación por parte de los que definen o proponen políticas o programas (Galaz et al., 2011).

En el enfoque renovador de formación orientado por la adquisición de competencias al que aluden Gervais y Desrosiers (2005), y que en gran medida comparto, éstas son definidas como un conjunto de recursos a movilizar en un contexto de acción profesional determinado. Agregan que, para el caso de la formación de profesionales de la enseñanza, tales recursos pueden ser conocimientos, habilidades/destrezas, actitudes (sociales y éticas), emociones (tensión, pasión) y otras competencias más específicas referidas por un ámbito de actuación o desempeño determinado de docencia. Por eso sigo cuestionando el excesivo énfasis que se ha puesto en la implementación chilena de políticas y dispositivos de formación y evaluación que se centran u orientan demasiado, y casi exclusivamente, a los recursos "cognitivos"; me animan también el reconocimiento y las propuestas que empiezan a surgir orientadas a una modalidad de mayor integralidad sistémica al respecto.

Otro elemento destacado por Gervais y Desrosiers (2005) para un enfoque más transformador es postular y concretar que las competencias/recursos no son puramente "personales/individuales" en su adquisición y desarrollo; también pueden ser sociales y compartidos con el medio laboral, académico y cultural (pares, redes, bancos de datos, publicaciones) y en diversas oportunidades y experiencias que es necesario aprender a valorizar (cursos, talleres, laboratorios, seminarios, visitas a terreno, las actividades mismas de "práctica", etc.).

Coincido con su postura sobre la consideración del contexto escolar como de aprendizaje de competencias múltiples y espacio propicio para la toma de conciencia y la identificación de necesidades en la variedad de competencias por parte de estudiantes y 
formadores. Pero suele acontecer que los profesores en formación (y también en ejercicio) tienen una relación "problemática" sobre su formación y suelen jerarquizar tales recursos en función de su utilidad para su actuación en el aula (lo que requerí para afrontar en un caso difícil no me lo enseñaron) o sin que valoricen los conceptos más teóricos de cursos y lecturas, que suelen ser menos perceptibles o pesquisables en las conductas del ejercicio docente ( $s i$ no fuera por las actividades en terreno... no se me aportó casi nada).

Sin duda que el acompañamiento reflexivo (Donnay y Charlier, 2006) por parte del o los formadores tiene máxima importancia en la adquisición de aprendizajes a partir de estas situaciones y problemas que se presentan en el ejercicio real de la enseñanza y son claves los procesos reflexivos que va logrando y compartiendo sobre su propia tarea de enseñanza.

En múltiples casos de mi actuación como colaborador académico de estudiantes en práctica inicial o de primeros años de docencia escolar me tuve que enfrentar a la alternativa de intentar cubrir o recuperar déficits formativos de contenidos o didácticos manifestados por ellos (tenerlo como objeto de una clase al respecto durante nuestros encuentros grupales o individuales), o aprovechar de generar un intercambio personal o grupal para reconocer y profundizar diferentes modalidades de intervención cuando tales situaciones se volvieran a presentar en el ejercicio de la enseñanza; el aprender a aprender solía producirse con mucha frecuencia. Mis propias reflexiones compartidas con otros formadores me fueron contribuyendo a optimizar ese camino.

Otro punto destacado por Gervais y Desrosiers (2005) apunta a la valorización de los saberes prácticos que fomentan adquirir las prácticas colaborativas en establecimientos escolares entre universidades y escuelas (en sentido amplio, establecimiento escolar de distintos niveles estructurales) al conocer y compartir dos aproximaciones distintas al conocimiento o saber docente. Si se explicitan y facilitan conjuntamente momentos de intercambios colaborativos entre el trío de actores, ello permite a los estudiantes ir definiendo y construyendo de modo paulatino y cada vez más autónomo su propia síntesis sobre la acción pedagógica, en el sentido que esta implica una relación dialéctica y constante entre teoría y práctica.

Sin entrar en mayor detalle y reconociendo múltiples diferencias a nivel estructural y administrativo, desde la presencia de una sola práctica larga y final en el programa formativo, se ha ido pasando a períodos más cortos integrados progresivamente en cada año del programa, hasta una práctica final integradora. En algunas experiencias, el proceso culmina con alguna "memoria" por escrito en que se identifiquen los aprendizajes conceptuales y pedagógicos o didácticos que el estudiante en formación destaca en ella y que debe discutir en un examen final, tras lo cual puede ser aprobado para la obtención de su título como profesor.

Sin duda, los discursos apuntan a elementos valiosos desde la perspectiva de mejoramiento conceptual, operativo y sistémico de las prácticas y de la formación inicial en su conjunto. Desde la literatura internacional y nacional y los medios de comunicación posibilitados por las nuevas tecnologías, así como en los contextos políticos, académicos, profesionales, estudiantiles y ciudadanos, se está destacando una creciente discusión sobre los marcos conceptuales que habría que apoyar y desarrollar; las políticas que habría que definir y dotar de recursos de toda índole (programas, estructuras, focos prioritarios en el sistema educacional en coherencia con el sistema social y cultural, rutas y plazos, recursos necesarios de diversa índole a considerar, proveer y supervisar); sobre los actores que debieran liderar las decisiones, su implementación y evaluación. Una perspectiva a tener en cuenta es que la investigación y evidencias con que se cuenta para abordar en concreto todas las dimensiones señaladas no son todavía todo lo abundantes ni coherentes que sería deseable. 
Estudios Pedagógicos, vol. XL, Número Especial 1: 239-256, 2014

PRÁCTICAS PROFESIONALES DURANTE LA FORMACIÓN INICIAL DOCENTE: ANÁLISIS Y OPTIMIZACIÓN DE SUS APORTES A LOS QUE APRENDEN Y A LOS QUE ENSEÑAN A APRENDER "A ENSEÑAR"

\subsection{ACTORES: SUS ROLES, COMPORTAMIENTOS Y DESARROLLO DURANTE LAS PRÁCTICAS PROFESIONALES}

Según Gervais y Desrosiers (2005), la consideración del contexto escolar como espacio de co-formación y de aprendizajes de todos los actores ha tenido repercusiones importantes respecto de la "misión" de la institución escolar, los roles jugados por el personal escolar y los propios estudiantes en práctica, y la colaboración del centro formador de educación superior con el establecimiento escolar. Seguiremos su secuencia y gran parte del contenido de su aporte, combinándolo con perspectivas similares o diferentes que otros autores presentan y con lo aprendido en mi propio desarrollo como formador.

\subsubsection{La misión del establecimiento escolar y sus actores}

Las autoras canadienses mencionadas resaltan que la misión "primaria" por parte de la escuela apunta a los aprendizajes y el desarrollo (integral) de sus propios alumnos. Como misión "secundaria" (no es necesario apreciarla como de menor rango o calidad), se espera que la escuela asuma (no sola) también la formación de estudiantes en práctica como una responsabilidad social de preparación de una nueva cohorte para una educación de calidad. Como cuestiones relevantes, es necesario preguntarse sobre las exigencias comprometidas en la articulación de ambas misiones.

El aumento del tiempo de presencia de los estudiantes en el establecimiento tiene fuertes incidencias sobre el trabajo cotidiano de todo el personal escolar: directivos, docentes y nodocentes administrativos y con otras labores. Sobre los dos primeros, existe relativamente mayor presencia de políticas, estudios, conocimientos y publicaciones acerca de diversas dimensiones de su actuación. Volveré más adelante a ese respecto.

Por mi parte, he optado por destacar algunos aportes adquiridos en mis actividades de participación en las prácticas con relación a los no-docentes. Los propios estudiantes me compartieron y enseñaron algunas actuaciones de tales actores que les habían ofrecido aprendizajes muy significativos para aterrizar su experiencia y conocimiento sobre el ejercicio docente. Menciono sólo algunas: acceso a reglas informales sobre conductas y relaciones con los distintos actores (incluyendo, además, a los apoderados); alternativas más eficientes y oportunas para acceder a la disponibilidad de recursos didácticos necesarios para su enseñanza, que frecuentemente escasean en la escuela, por los que hay cierta competitividad (tecnológicos, escritos); modos de acceso a ciertos elementos que resuelven necesidades más primarias (café y otros alimentos; medios de comunicación rápida interna y al exterior; espacios y momentos que facilitan reposo o descuelgues momentáneos en jornadas de mayor exigencia de clases. Es útil pensar que estas acciones, que podemos designar como propias de "un guía de visita", a veces son esperadas del aporte de los docentes o directivos, aumentando sus tareas lectivas, reconocidas como excesivamente recargadas si se consideran sus horas contratadas.

\subsubsection{Los estudiantes en formación inicial}

Una dimensión o arista que ha venido siendo bastante destacada en nuestro medio nacional refiere a la necesidad de discutir y reconsiderar los criterios existentes para la admisión de quienes postulan a seguir carreras de educación escolar en alguno de sus 
niveles escolares. Los que se aplican comúnmente en los últimos años han sido los puntajes obtenidos en las pruebas escritas de admisión a la universidad (PSU) y las notas conseguidas en la Educación Media, cuyo promedio permite otorgar a los alumnos una posición relativa de cada uno en los rankings elaborados.

No hay unanimidad de las instituciones de educación superior respecto a la aplicación y exigencia de tales instrumentos (un tercio de estudiantes entra a carreras de educación sin que se les exija rendir la PSU; sólo algunas se han aunado para emplear el Ranking), ni tampoco sobre el valor porcentual que asignan a cada instrumento en el conjunto del proceso de admisión. Aunque existen propuestas consideradas como avances, las discusiones sobre los criterios e instrumentos disponibles, su validación e incluso la aceptación de su real necesidad no se han agotado. Ella se ha asociado al debate acerca de los primeros proyectos sobre cambios en el sistema educativo formulados por el nuevo gobierno, específicamente respecto al que apunta a acabar con la selección de alumnos en el acceso a los procesos educacionales y formativos. Fundado en el principio de que la educación es un derecho básico de todo ciudadano y que este se ha estado impidiendo su cumplimiento y desarrollo mediante ciertas políticas y medidas aplicadas, el gobierno actual ha propuesto otras nuevas para intentar corregir progresivamente sus efectos de discriminación e inequidad, y producir mayor justicia, equidad e inclusión.

A modo de aproximación a ciertos focos en dichas discusiones, presento aquí un aporte de Herrera (2014), profesora chilena, quien sostiene dónde y cómo interesaría fomentar e implementar políticas y medidas que aseguren alguna "selección”, sobre la que, según ella, se requiere una necesaria discusión y consideración. La autora afirma que la carrera de formación pedagógica es determinante para el país y quien asuma esa responsabilidad no puede ser cualquier persona. Por ello, su postura apunta a que si los mecanismos formales de selección para ingreso a las carreras pedagógicas no se usan (1/3 de los admitidos lo hacen sin haber dado la PSU) o no se han validado plenamente respecto a lo que declaran identificar (la misma PSU sin cubrir todo lo que requerido para conocer integralmente a los candidatos), las falencias no justifican que se pueda interrogar, discutir y concordar la posibilidad de mecanismos complementarios que optimicen el acceso a la formación de los más preparados, aptos y dispuestos.

Como puntos a considerar y discutir, la profesora Herrera (2014) sugiere interrogarse sobre la necesidad de identificar cierto perfil especial y ciertas competencias fundamentales en el postulante, así como el manejo de una base disciplinaria mínima en contenidos a completar durante la formación. Como mecanismos o procedimientos complementarios sugiere el potencial aporte de las cartas de intención, las entrevistas o los tests psicológicos que han comenzado a utilizar algunas instituciones. Me ha resultado más concordante la valoración que ella hace al sugerir la extensión de los programas "propedéuticos", en la medida que combinan varios elementos que promueven ir avanzando en un sistema formativo más integral y progresivo: identifican más precozmente a personas con perfiles más "pre-preparados" o acordes con lo requerido en la carrera docente (conocimientos, cultura, motivación); se comprometen a un apoyo extra si se les produjeran mayores necesidades en formación disciplinares o de competencias de aprendizaje o relacionales. Resalta que dichos programas prefieren no privilegiar a los que se inscriben en carreras pedagógicas básicamente porque no les dio el puntaje para otras carreras, ni necesariamente trabajan con alumnos del más alto desempeño académico previo (PSU, notas de Educación Media). 
Estudios Pedagógicos, vol. XL, Número Especial 1: 239-256, 2014

PRÁCTICAS PROFESIONALES DURANTE LA FORMACIÓN INICIAL DOCENTE: ANÁLISIS Y OPTIMIZACIÓN DE SUS APORTES A LOS QUE APRENDEN Y A LOS QUE ENSEÑAN A APRENDER "A ENSEÑAR"

La propuesta recién presentada permite también formularme algunas interrogantes, entre otras sobre si tenemos ya suficientes conocimientos y evidencias respecto de los distintos elementos conceptuales y prácticos que subyacen a las modalidades en uso y sugeridas, por ejemplo en cuanto a las representaciones y comportamientos de los actores implicados, así como a las voluntades de algún cambio por parte de las autoridades de instituciones de formación, sobre todo en cuanto las propuestas les puedan implicar bastante complejidad en la obtención y el manejo de sus recursos.

Desde el enfoque de profesionalización progresiva del ejercicio de la docencia, es esperable que los estudiantes puedan exponer desde el inicio sus expectativas sobre las actividades de terreno y reajustarlas a medida del avance de estas; ello se facilita más si hay intercambios al respecto con sus formadores en los encuentros periódicos que poseen la mayor parte de los programas formativos. Reconociendo que existen importantes diferencias de exigencias, entre otras, en cuanto a contenidos y estructuras de los programas de formación de profesores para los distintos niveles del sistema escolar, comparten frecuentemente que el foco de tales encuentros se orienta a veces más a las dimensiones administrativas y de supervisión que caben a los formadores de la institución académica o del establecimiento escolar y preocupan también a los estudiantes; ello se complica más cuando la modalidad de contratación o dedicación efectiva de los formadores a las prácticas es "por horas".

\subsubsection{La formación de los formadores de futuros profesores}

En mi opinión, una consideración clave que no se puede eludir es que en nuestro medio existe notable carencia de políticas y programas integrales de formación de los formadores, así como de un cuerpo básico y variado de conocimientos acerca de éstos, que pudieran apoyar dichas finalidades. Solo desde mediados de la primera década de este siglo han ido apareciendo algunas voces de académicos y profesionales que han comenzado a señalar esta deficiencia y a postular medidas y esfuerzos que permitan resolverla oportunamente (Cornejo, 2005, 2007, 2011). Cabe señalar que la temática Formador de profesores (en adelante FP) ha dado origen a algunas publicaciones que relatan experiencias de profesionales de la formación docente (Cisternas, 2011; Fuentealba y Montenegro, 2011; Gajardo, 2011; Torres, 2011), y algunas instituciones universitarias han empezado a implementar unidades o programas de formación oficiales de sus docentes, tanto de Educación como de otras carreras o especialidades profesionales (entre otras, Universidad de La Serena; Pontificia Universidad Católica de Chile).

\subsubsection{Movimiento internacional sobre la profesión de formador de formadores}

Si se compara nuestro incipiente desarrollo de FP con el que llevan ya recorrido otros países que han liderado un verdadero movimiento orientado a la profesionalización de quienes se dedican a la formación del profesor (teacher educator), tenemos que ir decididamente a procesos que nos permitan mayores avances. En nuestro país se suelen proponer ejemplos de buenas prácticas formativas de otros países, especialmente fuera de América Latina, que han permitido a éstos lograr un éxito reconocido según ciertos indicadores; la indicación suele proponerlos como buenos referentes a tener en cuenta para nuestras políticas y prácticas. Yo he optado por algo similar al tomar como base de mi presentación, análisis 
y propuestas, una obra publicada recientemente por académicos holandeses (Lunenberg, Dengerink y Korthagen, 2014); a mi juicio, nos ofrece una perspectiva valiosa en la medida que presenta un panorama actualizado y seriamente fundado sobre el estado actual del conocimiento respecto a los FP. Las traducciones del inglés me pertenecen.

Los autores holandeses recuerdan una observación que hicieron Lanier y Little, en 1986, acerca de la limitada atención que se prestaba al importante trabajo de los FP y que su identidad, su actuación y su pensar eran frecuentemente mirados desde arriba en los estudios sobre formación docente (Lanier y Little, 1986 cit., en Lunenberg, Dengerink y Korthagen, 2014). Coincidente con la baja valoración del rol de los FP, al prologar y patrocinar la obra de Lunenberg, Dengerink y Korthagen, el académico australiano Loughran resalta que, al igual que la enseñanza, su trabajo ha sido superficialmente percibido como acción directa y fácil de entender; como consecuencia, el propósito de la formación de docentes, el conocimiento más complejo, las destrezas y habilidades requeridas para un buen desempeño han sido subvalorados o lamentablemente ignorados. Por ello el valor actual de este trabajo implica comenzar a articular aspectos cruciales de lo que significa ser un FP y crear oportunidades reales de una mejor comprensión de su relación con el desarrollo profesional de los mismos FP (Lunenberg, Dengerink y Korthagen, 2014: vii).

Volviendo al planteamiento de los autores holandeses, ellos agregan que, desde la década de los 90, un cambio fundamental ha tenido lugar y que el rol crucial de los FP en la cadena formativa gradualmente ha ido recibiendo mayor atención en la investigación, en la práctica y en la política educacional. Destacan que en la actualidad aparece un consenso casi general respecto a que, en un amplio grado, los FP determinan la calidad de los profesores y que éstos, a su vez, son un factor crucial para la calidad de la educación primaria y secundaria. De ahí surgiría la importancia de que los FP sean capaces de actuar a un alto nivel profesional.

A continuación, se interrogan acerca del significado que tiene la última afirmación del párrafo anterior y sobre si los FP cumplen ese requerimiento, reconociendo que la literatura especializada de los 90 no permitía aún dar una clara respuesta a las interrogantes; a pesar de su mayor frecuencia, aparecía como dispersa y no cumplía con la exigencia de una base teórica e investigativa compartida internacionalmente. Pero advierten también que algunas publicaciones de la última década (2010) han ofrecido respuestas parciales a temas sobre las características de la profesión y el comportamiento de los FP; de ahí que, si se apunta a tomar en serio la profesión de FP, la situación existente demanda un sólido análisis y síntesis sobre lo que se sabe en ese campo y ello fue el incentivo que los condujo a hacer un estudio de revisión al respecto y que constituye el objetivo y foco central de su publicación: un libro sobre los FP y su profesión.

El análisis y la síntesis de su revisión de literatura especializada sobre FP se orientó a responder qué roles profesionales eran identificables, qué factores críticos aparecían como determinantes de tales roles y su correspondiente comportamiento profesional (Lunenberg, Dengerink y Korthagen, 2014). Al explicitar su marco teórico, podemos aproximarnos al contenido de la expresión "FP" que he usado desde el inicio. Reconocen que los FP que se identifican con dicho término constituyen un grupo heterogéneo, por lo que se dificultan una descripción o definición que deje a todos satisfechos: se constatan diferentes antecedentes y experiencias docentes; diferentes contextos y niveles de actuación paralela o combinada, ya sea en instituciones académicas y/o escolares; diferentes tareas docentes a desempeñar: pedagógicas o disciplinares, acompañamiento en las prácticas; expectativas crecientes 
Estudios Pedagógicos, vol. XL, Número Especial 1: 239-256, 2014

PRÁCTICAS PROFESIONALES DURANTE LA FORMACIÓN INICIAL DOCENTE: ANÁLISIS Y OPTIMIZACIÓN DE SUS APORTES A LOS QUE APRENDEN Y A LOS QUE ENSEÑAN A APRENDER "A ENSEÑAR"

de actuaciones en formación continua e investigación. Dada la polisemia terminológica, los autores holandeses optan por una amplia e inclusiva definición: "todos los que, en instituciones académicas de formación docente o escolares, enseñan o acompañan a profesores en formación inicial, así como en su inicio o su continuidad en el ejercicio de la enseñanza" (Lunenberg, Dengerink y Korthagen, 2014: 5 y 63).

El rol profesional lo definen como "una interpretación personal de una posición, fundamentada en las expectativas del ambiente (lo que puede ser fuente de tensiones y conflictos) y en una base de conocimiento sistemáticamente organizada y transferible"; lo diferencian de la "identidad profesional", pues esta se focaliza más en las visiones personales y las imágenes de sí mismo. Para ellos, el "comportamiento profesional" refiere a una conducta fundamentada en una base de conocimiento sistemáticamente organizada y transferible, que expresa los valores y las normas de una comunidad profesional" (Lunenberg, Dengerink y Korthagen, 2014: 6 y 63; en esta última hay un error en la transcripción respecto a la primera).

Al explicitar y analizar críticamente la metodología que emplearon para revisar la literatura sobre FP (Lunenberg, Dengerink y Korthagen, 2014: 63-64; 9-18), señalan que se restringieron a artículos aparecidos entre 1991 y 2011 en publicaciones reconocidas por ISI y el Dutch Interuniversity Center for Educational Sciences. Una pesquisa inicial siguiendo los términos "teacher educator, teacher trainer y mentor teacher" les permitió identificar 1.260 publicaciones. Tras cotejarlos con su mayor pertinencia a los focos centrales de su pesquisa y una co-evaluación de jueces, optaron por 137 estudios relevantes, los que aparecen esquemáticamente presentados en un Apéndice del libro (Lunenberg, Dengerink y Korthagen, 2014: 108-159). La mayor parte de estos estudios se realizaron después del 2000 y se publicaron en Norteamérica (Estados Unidos y Canadá), Australia, Israel y algunos países europeos (Reino Unido y Holanda). Prevalecieron los cualitativos y de "menor escala"; el $58 \%$ emplearon metodologías de estudio de casos, self-studies y entrevistas. Los estudios cuantitativos fueron escasos y generalmente con muestras pequeñas. Las interrogantes formuladas fueron de tal diversidad, que les impidió realizar un meta-análisis.

\subsubsection{Panorama sobre roles, comportamientos y desarrollo profesional de formadores de profe-} sores

Al señalar los resultados sobre los focos investigados en su revisión de la literatura especializada de los últimos 20 años, Lunenberg, Dengerink y Korthagen (2014) señalan que han identificado seis roles característicos ejercidos por los FP que actúan en formación inicial (advierten que algunos roles figuraban juntos a otros, pero no se consideró para la sumatoria). A continuación los presento sintéticamente, indicando su frecuencia; su descripción y significación; factores críticos que se asocian a su adquisición, su comportamiento y desarrollo profesional, y propuestas básicas de los autores para su optimización.

\subsection{El FP como profesor de futuros profesores}

Constituyen el 48,9\% de los estudios analizados. Refieren a la formación inicial realizada en el contexto de una institución de educación superior y priorizan el comportamiento pedagógico del FP. En consecuencia, se reconoce la necesidad de conocimiento para 
adaptar su enseñanza al aprendizaje de sujetos adultos; por ejemplo para articular los conocimientos experienciales y teóricos en las prácticas.

También se reconoce la necesidad de competencias para promover un aprendizaje activo, autónomo y significativo; pero se agrega que su implementación no es fácil y a veces es inadecuada. Algo similar sucede con el reconocimiento que se da respecto del rol de "modelos" que deben ejercer los FP: exhibir buenos ejemplos de enseñanza efectiva, explicitar dicho comportamiento ejemplar y fundamentarlo teóricamente; reflexionar sobre sus sentimientos y explicitarlos para aportar al desarrollo afectivo de sus estudiantes. No obstante, en los estudios se reconoce también bastante dificultad para explicitar tal modelaje y una baja frecuencia de ejercicio.

En cuanto a factores críticos que faciliten el desarrollo y comportamiento profesional de este rol, se reconoce la existencia de un marco de referencia, por ejemplo respecto de estándares profesionales en cuya definición y evaluación participen los propios FP. En la misma publicación de Lunenberg, Dengerink y Korthagen (2014), se agrega un capítulo en que se realiza una presentación detallada del desarrollo de la Asociación de FP holandeses, que vendría liderando la mejora cualitativa de dicha profesión.

Otros factores críticos destacados en la literatura corresponden al reconocimiento de ciertas cualidades personales de los FP (deseo de aprender, interés en su campo disciplinar y en las características de sus estudiantes, fundamentados en el conocimiento y la experiencia existente). También se reconocen los beneficios que aportan el aprendizaje informal con los colegas, el acompañamiento de pares y las comunidades de aprendizaje; no obstante se señala la poca evidencia sistemática obtenida mediante estudios de seguimiento.

\subsection{El FP como investigador}

En un $19 \%$ de los estudios seleccionados se reconoce conceptualmente este rol. Se destaca un acuerdo internacional respecto que los FP lo desempeñen, pero en la práctica ello no es evidente y los FP resaltan las dificultades para su ejercicio. Existe poca claridad al respecto y necesidad de reconocerlo; mayor atención al tiempo y la información de que se dispone, así como sobre la provisión de un apoyo adecuado y la existencia de una cultura investigativa.

Uno de los focos de indagación más destacados en la literatura apunta a que los FP investiguen sobre su propia práctica de enseñanza. Los self-studies constituyen su principal modalidad de realización y se dispone de una abundante publicación sobre sus fundamentos conceptuales, sus finalidades y sus modalidades metodológicas (Cornejo, 2011; Loughran y Russell, 2002; Loughran et al., 2004; Pinnegar y Hamilton, 2009; Tidwell, Heston y Fitzgerald, 2009).

Como factores críticos que aporten al desarrollo y práctica del rol de investigador por parte de los FP se reconocen la creación de un contexto sistemático de soporte y colaboración, con una visión apropiada sobre la actividad investigativa y una explicitación clara de las expectativas y exigencias; también prestar atención al desarrollo de cualidades personales y de apertura sobre las posibles modalidades de indagación sistemática.

Otro factor, cuya potencialidad pude apreciar en mi actividad docente en formación inicial de inserción profesional y de postgrado, resalta la conveniencia de incentivos sistemáticos y significativos para iniciar y continuar las actividades de investigación, entre otros: publicación de la investigación, su informe a la propia institución de actividad docente, apoyo para presentación en congresos y seminarios. 
Estudios Pedagógicos, vol. XL, Número Especial 1: 239-256, 2014

PRÁCTICAS PROFESIONALES DURANTE LA FORMACIÓN INICIAL DOCENTE: ANÁLISIS Y OPTIMIZACIÓN DE SUS APORTES A LOS QUE APRENDEN Y A LOS QUE ENSEÑAN A APRENDER "A ENSEÑAR"

\subsection{El FP como colaborador en la formación inicial}

Este rol se explicita en el 10,9\% de la literatura especializada revisada. En su descripción se señala que refiere al proceso de apoyo (colaboración, coaching) a los estudiantes en formación tanto por el FP de la institución académica como el del centro escolar de práctica. Se reconoce un acuerdo general respecto a que se trata de un factor fundamental en la formación inicial de profesores en una perspectiva de profesionalización progresiva (ver también el punto 2.3.2.).

Por una parte, se señala un conjunto de tareas específicas respecto de este rol por parte del FP del centro escolar: no se trata de solo aconsejar e introducir a los estudiantes en las prácticas de centro escolar, sino también de plantearles interrogantes para la indagación, discusión y reflexión que se abra más allá del puro contexto escolar. Se reconoce también una escasa frecuencia práctica de esa perspectiva y de explicitación de su comportamiento docente y su concepción subyacente. El factor crítico que se destaca para la optimización de este rol refiere a la exigencia de una mejor preparación que apunte a una definición más amplia de su tarea.

Por otra parte, se señalan algunas tareas específicas respecto de este rol por el FP de la institución académica: necesidad de lograr un equilibrio adecuado entre su rol de apoyo y de formación (ver también Garant et al., 1999), así como diferenciarlos claramente del que corresponde al colaborador del centro escolar. También se menciona la necesidad de evitar un rol de enfoque terapéutico y de apuntar más a la discusión y reflexión crítica.

En la medida que la formación inicial docente aumenta su realización en colaboración y en el espacio mismo del ejercicio docente como factor crítico que contribuya a la optimización del ejercicio y desarrollo de estos roles complementarios, se precisa la necesaria implementación de una potente, sistemática e integral política y de programas con recursos variados y suficientes para llevarla a cabo; los de tiempo real de dedicación para los FP y la identificación y empleo de oportunidades existentes en ambos sectores formativos requiere también especial atención y decisión. Con ocasión del Proyecto de Fortalecimiento de la formación inicial docente (Ávalos, 2002), pude apreciar de cerca los beneficios para la implementación de prácticas de colaboración interinstitucional en acciones formativas de prácticas y de mentoría en algunos proyectos específicos (por ej. Cornejo y Padilla, 2008).

\subsection{El FP como constructor curricular}

Se identificó solo un $10 \%$ de estudios al respecto (aunque se advierte su eventual consideración en estudios sobre desarrollo curricular como tal). En general, se presentó un reconocimiento conceptual de este rol respecto al FP; no apareció nada sobre su desarrollo y comportamiento consecuente por parte de ellos.

Al analizar la ocurrencia de algún factor crítico para su optimización, se constata una variedad de principios orientadores que complican la opción de cada FP por este rol; además, este rol se ve determinado por circunstancias locales y discusiones sociopolíticas, En esa perspectiva, los FP son más bien seguidores que líderes. A pesar de una reciente mayor atención sobre el desarrollo curricular en la formación inicial de colaboración interinstitucional, una aproximación sistemática con propósitos específicos sigue escasa. 


\subsection{El FP como guardián del acceso a la profesión docente}

Una baja proporción de los estudios seleccionados para análisis $(5,8 \%)$ aborda este rol desde una perspectiva conceptual (en inglés se le menciona como gatekeeper), pero ninguno lo hace considerando su desarrollo y comportamiento en el ejercicio docente mismo. En general, los FP emplean estándares y perfiles que les fijan institucionalmente para evaluar a sus estudiantes, pero los caminos por los cuales pudieran alcanzarlos difieren bastante en los distintos contextos. Emplean usualmente los portafolios como modalidades de evaluación formativa y sumativa; pero como destacara en puntos anteriores, estas despiertan dudas y críticas sobre su validez y confiabilidad, y acerca de la misma evaluación de competencias en la medida que los contextos formativos e institucionales difieren en sus criterios sobre la calidad formativa. Una consecuencia reconocida refiere a las tensiones que experimentan los FP para equilibrar sus roles de colaboración y evaluación.

\subsection{El FP como agente animador de la colaboración formativa}

Un $8 \%$ de los estudios analizados lo describía conceptualmente; entre ellos solo uno había investigado su desarrollo y ejercicio en la enseñanza. Un mayor consenso sobre su importancia teórica en la formación inicial docente señala que su aporte esencial estaría en la creación de estructuras de cooperación de FP con distintos antecedentes y experiencias y ejerciendo la docencia en diferentes contextos, con la finalidad de mejorar la calidad de la formación de futuros profesores. En tal dirección, al animador y líder (en inglés broker) de la colaboración formativa le corresponde promover el desarrollo de una concepción, un enfoque y una identidad compartidos por el FP del centro escolar y de educación superior. Como competencias profesionales exigidas por su ejercicio se postulan las habilidades relacionales, así como las que lo capaciten para enfocar a los participantes en la atención a áreas específicas, a la consolidación de los resultados alcanzados y a una postura fundamentada en la investigación.

\section{CONSIDERACIONES FINALES}

Aunque todavía con algunas carencias en cuanto a volumen, expansión y divulgación internacional y a evidencias sistemáticas sobre lo que se declara discursivamente, las actividades de prácticas durante la formación inicial de profesores se han ido convirtiendo crecientemente en campo de reflexión sistemática y colaborativa de los propios FP. Es así que reconceptualizan sus supuestos y valores, sus roles y su ejercicio como profesionales de la enseñanza; establecen un progresivo sentido de comunidad de aprendizaje de y sobre la enseñanza, lo que les permite ir estableciendo relaciones dialécticas entre práctica y formación inicial docente e investigación de la enseñanza y formación inicial docente.

Complementariamente, la problemática de la Formación de los FP ha empezado también a reconocerse como foco y espacio específico y necesario de preocupación, análisis y propuestas que se implementan, aunque todavía con mayor frecuencia por parte de los responsables del desarrollo y gestión de procesos formativos en algunas instituciones de educación superior. Para avanzar más decididamente en la implementación de propuestas y programas de mejoramiento e innovación se requiere constituir progresivamente la 
Estudios Pedagógicos, vol. XL, Número Especial 1: 239-256, 2014

PRÁCTICAS PROFESIONALES DURANTE LA FORMACIÓN INICIAL DOCENTE: ANÁLISIS Y OPTIMIZACIÓN DE SUS APORTES A LOS QUE APRENDEN Y A LOS QUE ENSEÑAN A APRENDER "A ENSEÑAR"

Formación de FP en una función y un ámbito de institucionalización formal, no burocrática y participativa, así como de inversión de recursos específicos financieros (ítems para personal, gestión, espacios, investigación, publicación) y humanos (académicos con horas suficientes de dedicación y con formación especializada).

En lo que respecta a Chile, entre los proyectos del nuevo gobierno acerca de nuevas transformaciones políticas en el sector educacional se ha mencionado, aún en términos generales, uno que se focaliza en los "formadores". El alcance hasta ahora definido refiere mayoritariamente a los profesores en ejercicio: se reconoce su aporte esencial al mejoramiento de la calidad educacional en la perspectiva de superar la concepción de bien a adquirir y a implementar la de derecho ciudadano; la exigencia de un mejoramiento integral de su formación inicial y continua; la necesidad de una retribución económica más justa y de mejores condiciones laborales en su trabajo profesional. No hay duda que tales propósitos son valiosos y su implementación implicará esfuerzos y medidas que asegurarán su consecución.

No obstante, hago notar que ni por parte de las nuevas políticas gubernamentales comprometidas, ni por los movimientos gremiales de profesores, ni por los estudiantes secundarios y universitarios ni por los parlamentarios que inciden en las decisiones de políticas ha existido alguna mención expresa de los FP, tampoco de sus necesidades formativas iniciales (o de inducción) y de continuidad. Es posible que tal omisión corresponda a una poca especificación de lo implicado cuando se habla de una política orientada a la mejora de la formación inicial de profesores, más centrada, por ahora, en su estructuración y financiamiento y sin indicar específicamente lo referente a los actores implicados en su calidad formativa. Pero también pudiera coincidir con lo que en la revisión de la literatura internacional especializada apareció sobre el tiempo requerido para valorar mejor la profesión y el trabajo de enseñar a enseñar.

Para terminar, expreso una convicción compartida con otros colegas FP: las instituciones formadoras de nuevos profesores tienen especial responsabilidad en preparar nuevas generaciones de FP y generar nuevo conocimiento sobre cómo dotar de mejor calidad la formación inicial de profesores. Si las nuevas generaciones de FP se apropian de sus roles con conocimientos de lo que han aportado los estudios sobre formación inicial y con disposición y destrezas para estudiar su práctica formativa para mejorarla, los nuevos profesores y sus alumnos serán ciertamente los más beneficiados.

\section{REFERENCIAS BIBLIOGRÁFICAS}

Ávalos, B. (2002). Profesores para Chile, Historia de un Proyecto. Chile: Ministerio de Educación.

Cisternas, I. (2011). La experiencia de un trabajo de acompañamiento con profesores de Lenguaje y Comunicación: un análisis a partir de nuestras tensiones. En A. Galaz, R. Fuentealba, J. Cornejo y A. Padilla (Eds.), Estrategias reflexivas en la formación de Profesores y de Formadores de Profesores (pp. 221-235). Valdivia: Universidad Austral de Chile. Universidad Autónoma de Chile.

Cornejo, J. (2005). La formación de Formadores de Profesores: una deuda ineludible y un desafío pendiente. Foro Educacional, n.8, 15-45.

Cornejo, J. (2007). La formación de Formadores de Profesores: ¿para cuándo en Chile? 
Pensamiento educativo, vol.41, n.2, 37-55.

Cornejo, J. (2011). El self-study de la práctica de los formadores de futuros profesores: bases teóricas, características y modalidades metodológicas. En A. Galaz, R. Fuentealba, J. Cornejo y A. Padilla (Eds.), Estrategias reflexivas en la formación de Profesores y de Formadores de Profesores (pp. 115-174). Valdivia: Universidad Austral de Chile. Universidad Autónoma de Chile.

Cornejo, J. y Padilla, A. (2008). Reflexión y aprendizaje de la enseñanza entre profesores experimentados y principiantes. En J. Cornejo y R. Fuentealba (Eds.), Prácticas reflexivas para la formación profesional docente: ¿qué las hace eficaces? (pp. 55112). Santiago: Ediciones Universidad Católica Silva Henríquez.

Donnay, J. et Charlier, E. (2006). Apprendre par l'analyse des pratiques. Initiation au compagnonnage réflexif. Belgique: Editions du CPP, Presses Universitaire de Namur.

Feiman-Nemser, S. (2001). From preparation to practice: designing a continuum to strengthen and sustain teaching. Teachers College Record, vol.103, n.6, 10131055 .

Fuentealba, R. y Montenegro, H. (2011). Docencia en contextos de Educación Superior: la contribución de The Scholarship of teaching y la vinculación con los procesos reflexivos. En A. Galaz, R. Fuentealba, J. Cornejo y A. Padilla (Eds.), Estrategias reflexivas en la formación de Profesores y de Formadores de Profesores (pp. 175194). Valdivia: Universidad Austral de Chile. Universidad Autónoma de Chile.

Gajardo, M. (2011). Indagando en mi práctica docente. En A. Galaz, R. Fuentealba, J. Cornejo y A. Padilla (Eds.), Estrategias reflexivas en la formación de Profesores $y$ de Formadores de Profesores (pp. 195-207). Valdivia: Universidad Austral de Chile. Universidad Autónoma de Chile.

Galaz, A., Fuentealba, R., Cornejo, J. y Padilla A. (Eds.) (2011). Estrategias reflexivas en la Formación de Profesores y Formadores de Profesores. Valdivia: Universidad Austral de Chile. Universidad Autónoma de Chile.

Garant, C., Lavoie, M., Hensler, H. y Beauchesne A. (1999). El acompañamiento en la iniciación de la práctica docente: ¿invitación o freno a la emancipación? Boletín de Investigación Educacional, vol.14, 544-577.

Gervais, C. et Desrosiers, C. (2005). L'école, lieu de formation d'enseignants. Questions et repéres pour l'accompagnement de stagiaires. Canadá: Les Presses de l'Université de Laval.

Herrera, C. (2014). Educación: la selección que sí queremos. Centro de Investigación Periodística. CIPER. Recuperado desde http://ciperchile.cl/2014/06/09/ educacion-la-seleccion-que-si-queremos/

Imbernón, F. (1994). La formación y el desarrollo profesional del profesorado. Hacia una nueva cultura profesional. Barcelona: Grao.

Korthagen, F., Kessels, J., Koster, B., Lagerwerf, B., \& Wubbels, T. (2001). Linking practice and theory. The pedagogy of realistic teacher education. N. Jersey-London: Lawrence Erlbaum Associates, Publishers.

LEY 19.715. (2001). Otorga un mejoramiento especial de remuneraciones para los profesionales de la educación. Biblioteca del Congreso Nacional de Chile.

LEY 19.961. (2004). Sobre evaluación docente. Biblioteca del Congreso Nacional de Chile. 
Estudios Pedagógicos, vol. XL, Número Especial 1: 239-256, 2014

PRÁCTICAS PROFESIONALES DURANTE LA FORMACIÓN INICIAL DOCENTE: ANÁLISIS Y OPTIMIZACIÓN DE

SUS APORTES A LOS QUE APRENDEN Y A LOS QUE ENSEÑAN A APRENDER "A ENSEÑAR"

Loughran, J., Hamilton, M., LaBoskey, V., \& Russell, T. (2004). International Handbook of self-study of teaching and teacher education practices Chile. Dordercht. London. Boston: Kluwer Academic Publishers.

Loughran, J., \& Russell, T. (Eds.). (2002) Improving teacher education practices through self-study. London: Routledge/Falmer.

Lunenberg, M., Dengerink, J. \& Korthagen, F. (2014). The Professional Teacher Educator. Roles, Behaviour and Professional Development of Teachers Educators. Rotherdam. Boston. Taipei: Sense Publishers.

Ministerio de Educación. (2005). Informe Comisión sobre Formación Inicial Docente. Chile: Serie Bicentenario.

Pinnegar, S., \& Hamilton, M.L. (2009). Self-study of practice as a genre of Qualitative research. Dordrecht, The Nederlands: Springer Series.

Reyes, L., Cornejo, R., Arévalo, A. y Sánchez, R. (2010). Ser docente y subjetividad histórica en el Chile actual: discursos, práctica y resistencias. Polis, vol.9, n.27, 269-292.

Sisto, V. (2011). Nuevo profesionalismo y profesores: una reflexión a partir del análisis de las actuales políticas de profesionalización para la educación en Chile. Signo y Pensamiento, vol.31, n.59, 178-192.

Sisto, V. (2012). Identidades desafiadas: individualización, managerialismo y trabajo docente en el Chile actual. Psyché, vol.21, n.2, 35-46.

Tidwell, D., Heston, M., \& Fitzgerald, L. (Eds.) (2009). Research methods for the selfstudy of practice. Dordrecht, The Netherlands: Springer Series.

Torres, C. (2011). Indagando: autobiografía profesional. Cómo llegué a ser formadora de formadores. En A. Galaz, R. Fuentealba, J. Cornejo y A. Padilla (Eds.), Estrategias reflexivas en la formación de Profesores y de Formadores de Profesores (pp. 209220). Valdivia: Universidad Austral de Chile. Universidad Autónoma de Chile.

Van Manen, M. (2002). The pedagogical task of teaching. Teaching and teacher education, vol.18, n.2, 135-138.

Vaillant, D. y Marcelo, C. (2001). Las tareas del Formador. Málaga: Ediciones Algibe. 